\title{
Tele-Operated MRI-Guided Needle Insertion for Prostate Interventions
}

\author{
Pedro Moreira* ${ }^{* \|}$, Leanne Kuil*, Pedro Dias ${ }^{\dagger}$, Ronald Borra ${ }^{\ddagger, \S}$, \\ Sarthak Misra* \\ *Surgical Robotics Laboratory, Department of Biomechanical Engineering \\ University of Twente, The Netherlands \\ ${ }^{\dagger}$ Department of Biomechanical Engineering, Universidade Nova de Lisboa, Portugal \\ ${ }^{\ddagger}$ Faculty of Medical Sciences, Department of Nuclear Medicine and Molecular Imaging \\ University of Groningen and University Medical Center Groningen, The Netherlands

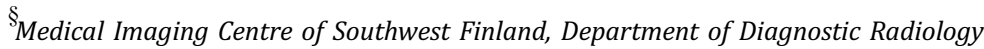 \\ Turku University Hospital, Turku, Finland \\ Surgical Robotics Laboratory, Department of Biomedical Engineering \\ University of Groningen \\ and University Medical Center Groningen, The Netherlands
}

Prostate cancer is one of the leading causes of death in men. Prostate interventions using magnetic resonance imaging (MRI) benefits from high tissue contrast if compared to other imaging modalities. The Minimally Invasive Robotics In An MRI environment (MIRIAM) robot is an MRI-compatible system able to steer different types of needles towards a point of interest using MRI guidance. However, clinicians can be reluctant to give the robot total control of the intervention. This work integrates a haptic device in the MIRIAM system to allow input from the clinician during the insertion. A shared control architecture is achieved by letting the clinician control the insertion depth via the haptic device, while the robotic system controls the needle orientation. The clinician receives haptic feedback based on the insertion depth and tissue characteristics. Four control laws relating the motion of the master robot (haptic device) to the motion of the slave robot (MIRIAM robot) are presented and evaluated. Quantitative and qualitative results from 20 human subjects demonstrate that the squared-velocity control law is the most suitable option for our application. Additionally, a pre-operative target localization algorithm is presented in order to provide the robot with the target location. The target localization and reconstruction algorithm are validated in phantom and patient images with an average dice similarity coefficient (DSC) of 0.78 . The complete system is validated through experiments by inserting a needle towards a target within the MRI scanner. Four human subjects perform the experiment achieving an average targeting error of $3.4 \mathrm{~mm}$.

Keywords: Minimally invasive surgery; prostate; MRI; biopsy.

Received 5 June 2017; Revised 28 January 2018; Accepted 12 February 2018; Published 9 April 2018. Published in JMRR Special Issue on Robotics-Assisted Needle Steering. Guest Editors: Mahdi Tavakoli, Sarthak Misra and Arianna Menciassi.

Email Address: "plopesdafrotamoreira@bwh.harvard.edu NOTICE: Prior to using any material contained in this paper, the users are advised to consult with the individual paper author(s) regarding the material contained in this paper, including but not limited to, their specific design(s) and recommendation(s).

\section{Introduction}

Prostate cancer is the second most common cancer in men worldwide. It is the fifth cause of cancer death in men [1]. Improvements in the diagnostic and treatment methods can reduce mortality rate of prostate cancer. The most common methods for diagnosis are the prostate-specific antigen (PSA) test and the digital rectal 
examination (DRE). If there is an indication of cancer, a prostate biopsy is done to confirm the diagnostic. Prostate biopsies are usually guided by transrectal ultrasound (TRUS) images. However, early stage lesions might not be visible in the conventional ultrasound (US) images [2]. If the suspected lesion is not correctly targeted, the procedure may result in a false diagnosis.

A solution for accurate prostate biopsies is the use of magnetic resonance (MR) imaging. MR images have higher tissue contrast and larger spatial resolution than US images. Abnormalities in the prostate tissue can be detected in MR images, indicating the location of the suspected tumor. However, MR-guided prostate biopsy is a time-consuming procedure and its costs are relatively high if compared to TRUS-guided biopsy. In the guideline proposed by Barentsz et al., patients are only subjected to an MR-guided biopsy after incongruent results of PSA test and TRUS-guided biopsy [2]. Besides the improvements to the clinical outcomes, the use of robotic systems can facilitate MR-guided prostate interventions and increase its clinical indication. The Minimally Invasive Robotics In An MRI environment (MIRIAM) robot aims to facilitate MR-guided prostate interventions [3].

The MIRIAM robot is an MR-compatible robot that combines piezoelectric and pneumatic actuation methods to achieve a precise prostate biopsy (Fig. 1). The system integrates pre-operative path planning and needle steering algorithm. The robot is able to perform a fully autonomous biopsy. However, autonomous systems are still not widely accepted by the clinical community [4]. Several robotics developers consider important to

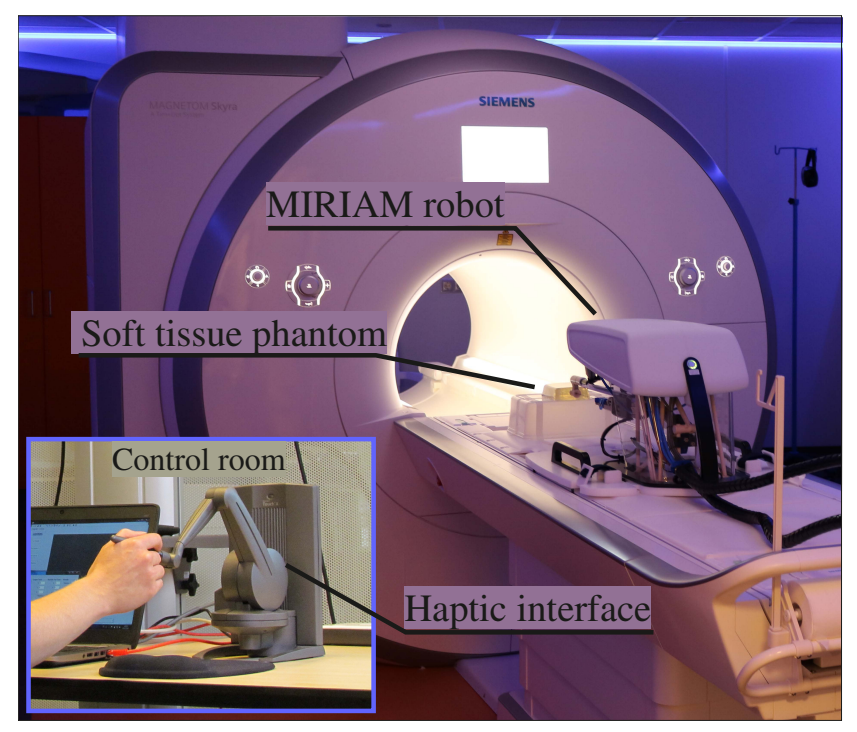

Fig. 1. The MIRIAM robot is Magnetic Resonance (MR)-compatible system designed for needle-based interventions in the prostate. The system is capable of inserting, rotating and firing a biopsy needle to collect tissue samples. In the current implementation, the user controls the insertion using a haptic device placed at the control room. provide the clinician with control of the procedure. Even in commercially-available robotic systems which can operate autonomously (i.e. the ROBODOC), there are concerns over accepting autonomous modes [5]. Therefore, it is essential to provide the clinician control of the most critical actions of the robot during the procedure. This work aims to allow the clinician to control the procedure by implementing an user interface for teleoperating the MIRIAM robot during needle insertion.

\subsection{Previous work}

Several surgical robotic systems which includes the clinician in the control loop have been presented. Hungr et al. proposed an autonomous system for needle-based interventions that can switch to a manual operation mode in case of emergency conditions [6]. Piccin et al. presented a CT-compatible needle driver that grasps and inserts the needle, mimicking the surgeon gesture [7]. The driving mechanism includes a force sensor to measure the insertion force and provide feedback to the clinician. Majewicz et al. proposed a system where the user tele-operates the movement of a flexible needle using a haptic device [8]. The force feedback is used to provide the kinematic constraints of the flexible needle. Romano et al. presented a comparison between openloop and tele-operated flexible needle steering using three different control laws [9]. The comparison showed that a hybrid control law resulted in best targeting accuracy. Later, Abolhassani et al. presented a tele-operated system for needle insertion using force feedback and US images to monitor the insertion [10]. A comparison between autonomous, teleoperated and semi-autonomous needle insertion was also presented. Zarrad et al. also proposed a tele-operated needle insertion using force feedback [11]. The system had a conventional force sensor and was controlled using state feedback estimated by an Active Observer.

Abayazid et al. presented a tele-operated needle steering system that provides visual feedback as well as vibratory feedback [4]. The user controlled the needle rotation, but the insertion velocity was kept constant for the entire experiment. Although the system was able to achieve a high accuracy, the user was controlling the needle rotation and not the insertion depth, which reduces the controllability of the system.

The combination of robotic actuation and manual needle insertion has been widely implemented in robots for prostate interventions. Wei et al. presented a comanipulated system where the user controlled the needle insertion into the prostate [12]. The system is composed of a robotic arm able to position the needle guide for prostate brachytherapy allowing the user to insert the needle manually. Similar approaches have been used for MR-guided prostate biopsies. Schouten et al. 
developed a pneumatic positioning device for MR-guided transrectal prostate biopsy [2]. Stoianovici et al. presented an MR-compatible system for endorectal prostate biopsy composed of a passive arm and an actuated 3 degrees-of-freedom (DoF) end-effector [13]. However, these robotic systems presented errors associated with the manual needle manipulation. Moreover, manual insertion requires the clinician to cope with the space constraints of the MR bore or the removal of patient from the bore.

Although studies with tele-operated needle insertion have already been published, just a few tele-operated systems for MR-guided needle-based procedures have been introduced. Goldenberg et al. presented a teleoperated robot for MR-guided interventions in the prostate [14]. The robot is controlled through a joystick without force feedback located in the control room. Seifabadi et al. demonstrated the feasibility of using fiber Bragg grating (FBG) sensors to measure the force and provide force feedback to the user operating an MR compatible robot [15]. The most important challenge towards force feedback in MR-guided tele-operated interventions is the lack of a reliable MR-compatible force sensor. Analytical needle-tissue interaction models can be used to estimate the exerted force and provide the user with haptic feedback. The accuracy of the force estimation is not critical if the force information provides haptic perception for the user and does not directly control the robot. Several force models for needle insertion have already been proposed. Okamura et al. proposed a needle insertion modeled by adding stiffness, friction and cutting forces [16]. Barbé et al. presented a needle-tissue interaction force model based on the Kelvin-Voigt model [17]. In their work, the parameters of the model are estimated using a force sensor attached to the needle base. Kobayashi et al. proposed a model for insertion forces in an in-vitro liver based on fractional derivatives [18]. A complete review of haptic feedback in needle insertions was presented by Ravali and Manivannan [19]. This work aims to develop a tele-operated system with force feedback using an interaction needletissue model defined by the stiffness of the soft-tissue (Young's modulus). The user controls the insertion depth of a biopsy needle, while the MIRIAM robot controls the orientation to insert the needle towards a suspected lesion. The suspected lesion is considered the target, and therefore defining its exact location is crucial for an accurate procedure.

Target localization in prostate interventions is a challenging task. Although there has been extensive research in segmenting particular structures of the human body [20-23], a prevalent effort in analyzing and segmenting a small region of interest suspected to contain cancerous cells is fairly in more recent [24] research ethics committee prior to being conducted. A state-of-art review was presented by Wang et al. [25]. Most of the methods for automatic prostate cancer segmentation resort to Machine Learning algorithms, such as the use of a pixel-wise Bayesian classifier in a multi-resolution scheme [24]. Ozer et al. presented a method for segmenting the suspected region with multispectral MRI using both a supervised and an unsupervised learning method [26]. Guo et al. presented a deformable MR prostate segmentation method by unifying deep feature learning with the sparse patch matching [27]. Moreover, the use of Support Vector Machines (SVM) with Conditional Random Fields has been reported to have an increased accuracy in delineating the region of interest [28]. The use of Markov Random Fields, coupled with multispectral MRI, has also been proposed for prostate cancer segmentation [29]. Gabor filtering-based analysis [30] and the wavelet-based SVM have also been used on prostate research to segment the gland or produce a diagnosis [31]. Most of these studies depend on a specific MRI protocol and require intensive computational resources. Signal intensity is not standardized and acquisition protocol, field strength, coil profile and scanner type greatly affect the image appearance and the performance of the segmentation [32]. In this work, we propose an algorithm to localize and reconstruct the suspected lesion using a sequence of MRI slices and basic image processing techniques. The algorithm is used in the pre-operative planning to define the target location for the tele-operated needle insertion.

\subsection{Contributions}

This paper presents a tele-operated robotic system using a 9 DoF robot system for MR-guided transperineal prostate biopsy. The user tele-operates the MIRIAM robot using a haptic device. To overcome the challenges of placing a force sensor within the MR bore, a needletissue interaction force model is developed and used to provide haptic feedback to the user during the insertion. The model uses biomechanical information (stiffness) of the soft tissue estimated non-invasively by Acoustic Radiation Force Impulse (ARFI) technique. Four well-known control laws to relate user input into robot commands are quantitatively and qualitatively evaluated in human subject studies. Different from previous studies, our comparison also includes a qualitative assessment using the user's opinion to define the best control law for the MIRIAM robot. In addition, we also present an algorithm to localize the suspected lesion in MR images. The algorithm is based on two different techniques and it is validated in phantom and patient images. The algorithm provides the target position for the robot-assisted procedure. Moreover, the algorithm is able to define the shape of the target. This information is important during the pre-operative planning to maximize the amount of tissue sample collected by the needle. The proposed 
overall system is evaluated through experiments into soft tissue phantoms inside a 3T MRI scanner.

\section{Material and Methods}

This section describes the robotic system, the force interaction model, the tele-operated architecture. The target localization algorithm used in the pre-operative planner is also presented.

\subsection{MIRIAM robotic system}

The MIRIAM robot is an MR-compatible system that uses piezoelectric and pneumatic actuation to insert and fire a biopsy needle. The robot has five rods with adjustable length to provide translational and rotational motion for the needle driver. The needle driver rotates and inserts the needle into the prostate. Moreira et al. previously described the design and autonomous operation of the robot [3]. The needle steering capability and the MRcompatibility of the robot were evaluated in a MAGNETOM Aera scanner. In our previous work, a biopsy needle was steered towards a target location defined by the user. In this work, we included the robot in a tele-operated system. The MIRIAM robot is the slave robot, while the Geomagic Touch X (3D Systems, USA) is the master robot. The master robot is used to control the insertion depth and it is able to provide force feedback to the user.

\subsection{Force feedback modeling}

Forces exerted on the needle during insertion $\left(f_{\mathrm{n}}\right)$ are due to puncture $\left(f_{\mathrm{p}}\right)$, friction $\left(f_{\mathrm{f}}\right)$ and cutting forces $\left(f_{\mathrm{c}}\right)[16]$ :

$$
f_{\mathrm{n}}=f_{\mathrm{p}}+f_{\mathrm{f}}+f_{\mathrm{c}} .
$$

In transperineal prostate biopsies, the puncture forces can be neglected, since the perineum is preloaded and the puncture occurs just after the insertion starts. In several friction models, such as the Coulomb-viscous model, the friction forces depend on the velocity [33]. However, during the needle insertion, contact surface varies as the needle is inserted. Therefore, we propose a model where the friction depends on not only the velocity but also on the insertion depth, such as

$$
f_{\mathrm{f}}(\dot{x}, x)= \begin{cases}k_{1}+k_{2} \dot{x} x, & \dot{x}>0 \\ 0, & \dot{x}=0 \\ k_{3}+k_{4} \dot{x} x, & \dot{x}<0,\end{cases}
$$

where, $x$ and $\dot{x}$ are the insertion depth and velocity, respectively. The constants $k_{1}, k_{2}, k_{3}$ and $k_{4}$ are estimated in Sec. 3.1.
Besides the friction, the cutting force is an important component of the total exerted force, as described in Eq. (1). Ideally, the cutting force should be constant and dependent on the tissue characteristics. However, the cutting force increases with the insertion depth $(x)$ due to the compression of the tissue and needle curvature. Therefore, the cutting force is modeled considering the insertion depth $(x)$ as well as Young's modulus $(E)$ of the tissue and needle curvature $(\kappa)$ :

$$
f_{\mathrm{c}}(x, \kappa, E)=k_{5} x E+k_{6} \kappa .
$$

Combining (2) and (3), the needle-tissue interaction force model is given as

$$
f_{\mathrm{n}}(\dot{x}, x, E, \kappa)= \begin{cases}k_{1}+k_{2} \dot{x} x+k_{5} x E+k_{6} \kappa, & \dot{x}>0 \\ 0, & \dot{x}=0 \\ k_{3}+k_{4} \dot{x} x . & \dot{x}<0 .\end{cases}
$$

The needle curvature is directly related to the tissue stiffness, therefore the online estimation of the needle curvature can also capture changes in the tissue stiffness during the insertion. The presence of the Young's modulus in our model together with the online curvature estimation allow us to correct possible modeling errors. Additionally, the model can be combined with different force sensing techniques to provide haptic feedback during insertions into unknown or inhomogeneous tissues. The proposed model can also be adapted to represent nonlinear tissue properties. The model parameters are estimated based on the force information acquired in a series of insertions into different phantoms and presented in Sec. 3.1. The identified model is implemented in the tele-operated system.

\subsection{Tele-operation architecture}

The overall control architecture of the proposed teleoperated system is presented in Fig. 2. The user controls the needle insertion using the haptic device (master robot). The master controller is based on the standard force control library provided by the haptic device manufacturer with a refresh rate of $750 \mathrm{~Hz}$. Motion in $x$ and $y$-axes are counteracted by virtual fixtures to keep the user within the insertion axis [34]. Different control laws can be used to transform the output of the haptic device into insertion commands for the robot. Four possible control laws are selected to be tested in the MIRIAM robot:

(1) Position control law: $x_{s}=k_{p} x_{m}$.

(2) Velocity control law: $\dot{x}_{s}=k_{v} \dot{x}_{m}$.

(3) Squared velocity control law: $\dot{x}_{s}=k_{s} \dot{x}_{m}^{2}$.

(4) Damper control law: $\dot{x}_{s}=\left(1 / k_{d}\right) f_{m}$.

The position control law relates the position of the haptic device, which is the master robot $\left(x_{m}\right)$, to the position of 


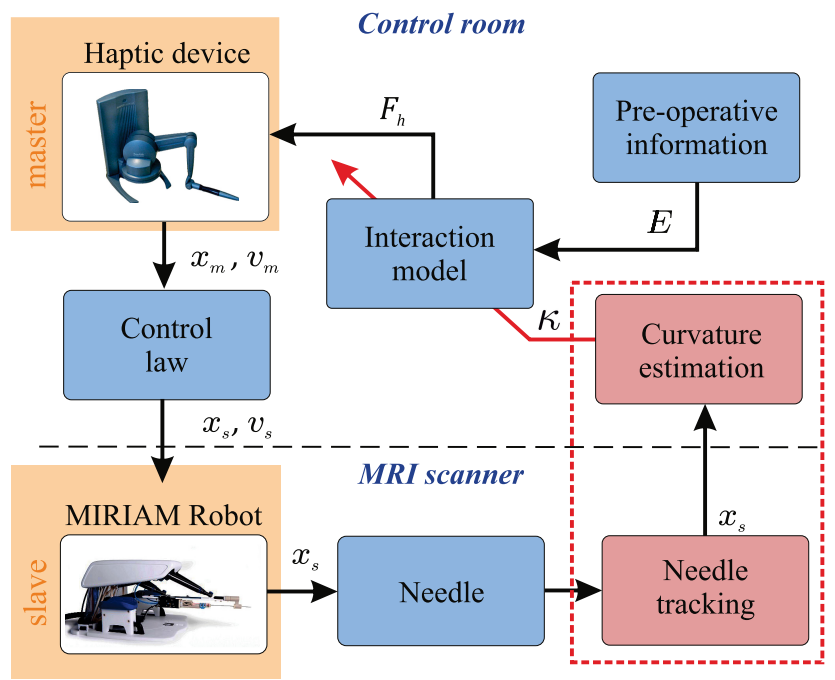

Fig. 2. Overall tele-operation architecture of the MIRIAM robot. The user controls the needle insertion through a haptic device, which provides the master position $\left(x_{m}\right)$ or master velocity $\left(v_{m}\right)$. The slave velocity $\left(v_{s}\right)$ and position $\left(x_{s}\right)$ are calculated based on the control law. A needle tip tracking using fiber Bragg grating sensors estimates the needle tip position and calculates the needle curvature $(\kappa)$. The needle curvature and the Young's modulus of the tissue $(E)$ and the needle deflection provides the force feedback $\left(F_{h}\right)$ to the user.

the MIRIAM robot, which is the slave robot $\left(x_{s}\right)$, using a scaling term $\left(k_{p}\right)$. The value of $k_{p}$ is a tradeoff between the range of motion and accuracy, the value is defined as $k_{p}=\frac{x_{s, \max }}{x_{m \text { max }}}$, which gives a $k_{p}$ of 0.95 . In the velocity control law, the master velocity $\left(\dot{x}_{m}\right)$ is translated to the slave velocity $\left(\dot{x}_{s}\right)$ using scaling term $k_{v}$. The gain $k_{v}$ was experimentally tuned to 0.5 . The squared velocity control law is similar to the velocity law, but using the master velocity squared and the scaling term $\left(k_{s}\right)$. The value of $k_{s}$ is set such that the maximum slave velocity is reached when the master velocity is twice the maximum slave velocity, giving a value of 25 . In the damper control law, the force exerted on the master robot by the user $\left(f_{m}\right)$ is transformed into slave velocity $\left(\dot{x}_{s}\right)$ using a virtual damper coefficient $\left(k_{d}\right)$. The damper coefficient is tuned such that the maximum velocity of the slave robot $(0.01 \mathrm{~m} / \mathrm{s})$ is set at two-thirds of the master range of motion, resulting in a value of $k_{d}$ of 269 . The most suitable control law for the MIRIAM robot is selected based on human subject experiments presented in Sec. 3.2. The best control law is used in the experiments inserting a needle towards a target within the MRI scanner.

\subsection{Pre-operative target localization}

This section presents the image processing algorithm used to segment the suspected lesion and define the target location. The algorithm segments a suspected lesion and reconstructs the 3D shape of the target. Before the segmentation starts, the user (clinician) defines in which region of the prostate suspected lesion is located. The algorithm provides the center and the shape of the lesion, which are important to maximize the amount of collected tissue sample.

The segmentation is comprised of two fundamental sub-algorithms. The first sub-algorithm uses the pixel intensity at the center of region of interest (ROI) and separates the remaining pixels into two distinct groups: (i) a group containing the pixels whose intensity is closer to the aforementioned central value; (ii) a group containing pixels whose intensity values are further than the central value. Thus, the sub-algorithm defines in which group the pixels have to be classified. The classification is such that it maximizes the inter-class variance of both groups, which is a procedure similar to Otsu's standard method for automatic thresholding [35]:

$$
\sigma_{T}^{2}=\omega_{1}(t) \omega_{2}(t)\left[\mu_{1}^{2}-\mu_{2}^{2}\right],
$$

where $\sigma_{T}^{2}$ is the inter-class variance, $\omega_{1}$ and $\omega_{2}$ are the probabilities of a randomly chosen pixel to belong to one class or the other, while $\mu_{1}$ and $\mu_{2}$ are the pixel intensity averages for each class. The threshold which maximizes the intra-class variance is chosen and the pixels which are marked as closer to the center value are considered as part of the target (Fig. 3).

The second sub-algorithm focuses on the edge detection rather than the region detection. It considers the same region defined by the user and its purpose is the detection of pixels where the change in contrast is the greatest.

The sub-algorithm draws a set of lines passing through the center of the ROI with an equal angular distance between them. The lines are divided into two segments and the highest derivative of each segment is defined as the sharpest edge (Fig. 3). The pixels representing the edges of the target are connected using the Bresenham's method and the inner portion of the polygon is filled. The result is considered the suspected lesion by the edge-based algorithm. A pixel is considered part of the suspected lesion (target) if detected by both algorithms (Fig. 3). The process is repeated for each image slice and the 3D shape of the suspected lesion is reconstructed using the contour points of the segmented images. The algorithm is validated using a set of MR images of gelatin phantoms, biological tissue and patient data.

\subsection{MRI experiments}

The feasibility of using the tele-operated robot within an MRI scanner is demonstrated by experiments conducted in the Siemens Magnetom Skyra MR-scanner (Siemens AG, Germany).

The subject controls the robot using the haptic device placed inside the control room. Real-time MR images are 


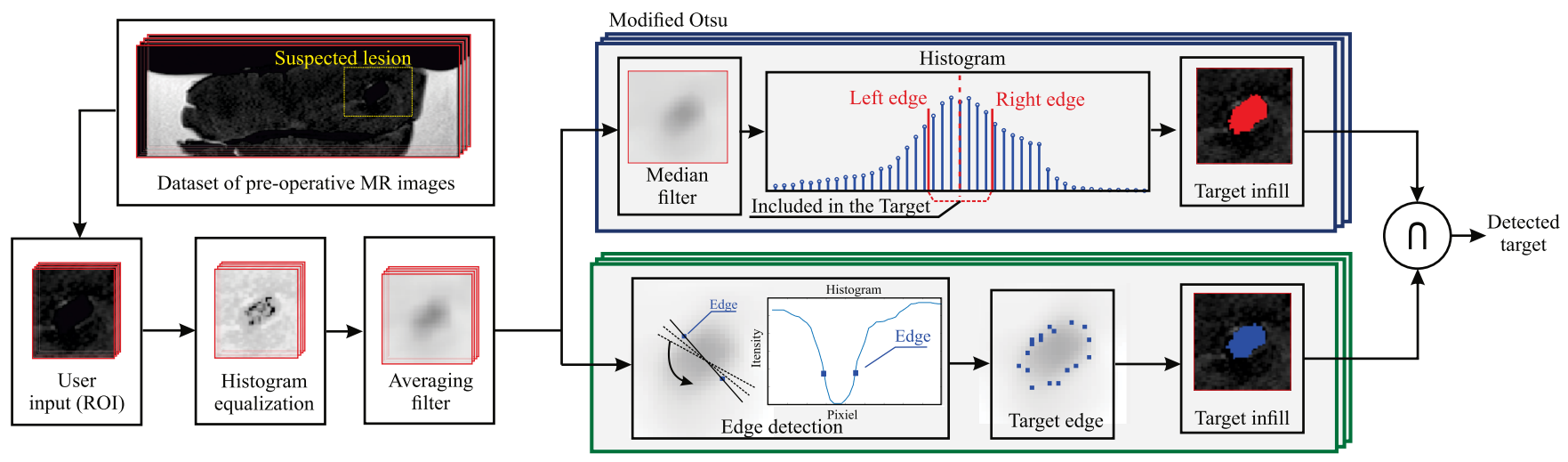

Fig. 3. A diagram representing the algorithm for segmenting the suspected lesion. The slices are subjected to a pre-processing phase, where the image is filtered and equalized. Two methods of segmentation are applied: one region-dependent and the other edge-dependent. The region-dependent algorithm is based on Otsu's method, while the edge-dependent uses the highest derivatives of multiple segments to trace the suspected lesion contour. The segmented regions are subjected to an AND logical operation ( $\cap$ ). Pixels approved by both algorithms are included in the final segmented lesion and considered as the detected target.

presented to the subject in order to supervise the insertion. The robot inserts a clinically approved biopsy needle (MR-Clear Bio-Cut, Sterylab, Italy) using the squared velocity control law. The soft tissue phantom is made of gelatin with a Young's modulus of $45 \mathrm{kPa}$ and has two embedded targets. The targets are spheres with $1.5 \mathrm{~mm}$ radius made of PVC. The target locations are defined using the algorithm presented in Sec. 2.

A path planner uses the target locations to determine the best needle entry point and insertion angle. The planner determines the shortest path direction connecting the insertion region and the target. The needle path is then traced along the path direction using a needle deflection model. If the insertion environment contains obstacles or no-go zones, the algorithm rotates the original path about the vertical axis. The planner defines the insertion location and angle for each target location. For more details on the path planner, we refer the reader to our previous study [3].

\section{Results}

This section first presents the experimental results of the model identification and the evaluation of the best control law for the MIRIAM robot. The evaluation of the target detection and experiments inside the MRI scanner are also presented.

\subsection{Force model identification}

A force sensor (ATI Nano-17, Industrial Automation, USA) is attached to a needle in order to collect force measurements during the insertion (Fig. 4). The experiments are performed with a needle integrated with an array of 12 FBG sensors, divided along three optical fibers. The needle has a diameter of $1.2 \mathrm{~mm}$ and a bevel tip angle of $60^{\circ}$. The FBG sensors are used to estimate the needle curvature $(\kappa)$ [36]. The needle is inserted into soft tissue phantoms using the MIRIAM robot. The phantoms are prepared with a mixture of water, gelatin and silica to mimic the stiffness of prostate tissue.

\subsubsection{Friction force}

The experiments to identify the friction parameters of (2) are performed in three different gelatin phantoms with a mass ratio of $5 \%, 7.5 \%$ and $10 \%$ gelatin and $1 \%$ silica. Although artificial phantoms are intrinsically different from real tissues, gelatin phantoms have been widely used in needle insertion experiments to mimic real tissues and demonstrate feasibility. These gelatin concentrations resulted in Young's moduli $(E)$ of 11, 34 and $69 \mathrm{kPa}$, respectively. These values are within the range of the Young's moduli found in the literature for prostate and surrounding tissues [37]. The phantom's mass, dimensions and shear wave velocity are used to define the Young's modulus [38]. The shear wave velocity is acquired non-invasively with ARFI available on the Siemens AcusonS2000 US machine (Siemens AG, Germany).

The phantoms are cut into different pieces with thicknesses of 20, 30 and $40 \mathrm{~mm}$. Before each experiment, the needle is inserted through the entire phantom to avoid cutting forces (Fig. 4(a)). Maximum velocity is varied between $1 \mathrm{~mm} / \mathrm{s}$ to $15 \mathrm{~mm} / \mathrm{s}$ and a total of 63 experiments are performed. The force measurements are fitted with least-squares linear regression to the friction force model. The least square problem is modeled as: $\mathbf{f}_{\text {forces }}=\mathbf{A p}$, where $\mathbf{f}_{\text {forces }}$ is a vector composed by all $N$ 


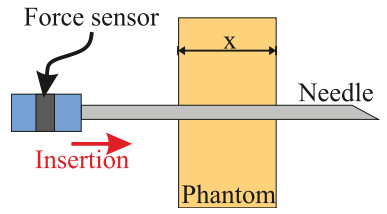

(a)

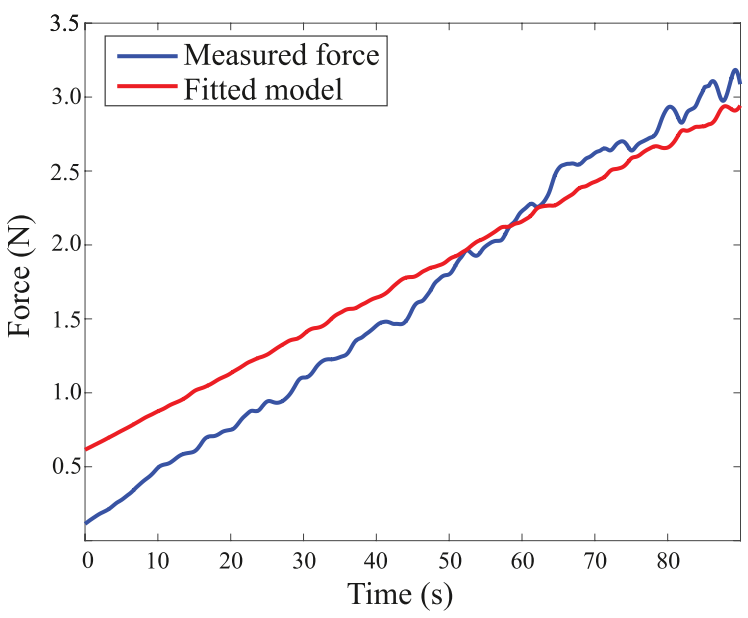

(c)

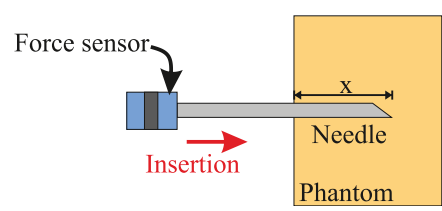

(b)
Fig. 4. (a) Friction force modeling experiments: Phantoms with different thickness $(x)$ are used to identify the friction force depending on the insertion depth. (b) Cutting force modeling experiments: The needle is inserted into a phantom. The friction force, based on insertion depth $x$, is subtracted to determine the cutting force. (c) Cross-validation: Measured force and the force estimated by the fitted model (friction and cutting force) of one insertion experiment.

force measurements, $\mathbf{A}$ is the $N$ by $n$ measurement matrix ( $n$ is the number of parameters to be estimated), and $\mathbf{p}$ is the vector with $n$ parameters. The fitted model is then defined as follows:

$$
f_{\mathrm{f}}(\dot{x}, x)= \begin{cases}0.70+0.00057 \dot{x} x, & \dot{x}>0 \\ 0, & \dot{x}=0 \\ -0.54+0.0012 \dot{x} x, & \dot{x}<0 .\end{cases}
$$

\subsubsection{Cutting force}

Three gelatin phantoms are used with Young's moduli (E) of 13,34 and $58 \mathrm{kPa}$. The insertion velocity is varied between $1 \mathrm{~mm} / \mathrm{s}$ to $15 \mathrm{~mm} / \mathrm{s}$, and insertions are done from 0 to $90 \mathrm{~mm}$ (Fig. 4(b)). A total of 21 cutting force measurements are performed. The force measurements are fitted to the cutting force model (3) using leastsquares linear regression. The fitted model is then defined as follows:

$$
f_{\mathrm{c}}(x, \kappa, E)=0.00043 x E-106 \kappa \text {. }
$$

Combining (6) and (7), the complete needle-tissue interaction force model is then given by

$$
\begin{cases}f_{\mathrm{n}}(\dot{x}, x, E, \kappa)=0.70+0.00057 \dot{x} x & \\ \quad+0.00043 x E-106 \kappa, & \dot{x}>0 \\ 0, & \dot{x}=0 \\ -0.54+0.0012 \dot{x} x . & \dot{x}<0 .\end{cases}
$$

\subsubsection{Cross validation of the force estimation}

The fitted model is validated using a new set of force measurements during needle insertion. The proposed model is also compared with well-known interaction force models, such as the Kelvin-Voigt and the elastic model [39]. The parameters for each model are estimated using one dataset, and the force estimation is compared to a new dataset of measurements. The new dataset is composed of five insertions performed in different phantoms. The root mean square error of all measurement points is used to evaluate each model, such as:

$$
\mathrm{RMSE}=\sqrt{\frac{1}{N} \sum_{i=1}^{N}\left(f_{e}(i)-f_{m}(i)\right)^{2}},
$$

where $f_{e}$ is the estimated force, $f_{m}$ is the measured force and $N$ is the total number of measurements. The RMSE for each model is presented in Table 1. The proposed model presents the lowest force error among the analyzed models. The measured force and the force estimated by the fitted model of one representative experiment are presented in Fig. 4(c). The proposed force model is then used to provide force feedback during the tele-operation of the MIRIAM robot.

\subsection{Tele-operated evaluation}

Human subject experiments are performed to determine the most suitable control law for the MIRIAM robot. A total of 20 human subjects (22-36 years old) performed the experiments, of which 13 males and 7 females. The

Table 1. The root mean square error between measured and estimated forces of the proposed model and well-known models.

\begin{tabular}{lc}
\hline Model & Root mean square error \\
\hline Spring & $0.66 \mathrm{~N}$ \\
Spring-damper & $0.57 \mathrm{~N}$ \\
Kelvin-Voigt & $0.60 \mathrm{~N}$ \\
Proposed model & $0.41 \mathrm{~N}$ \\
\hline
\end{tabular}


subjects were mainly senior biomedical engineering students. The subjects participated on a voluntary basis and were informed about the procedure before the beginning of the experiment. A familiarization period was also provided to make them acquainted with the experimental setup. A biopsy needle (MR-Clear BioCut, Sterylab, Milan, Italy) is used in the experiments for clinical relevance. It has the same geometrical properties as the FBG-needle, but is less flexible and therefore the curvature $(\kappa)$ is considered zero in the current experiments.

Each subject performs eight needle insertions into a gelatin phantom, reaching an insertion depth of $70 \mathrm{~mm}$. The order of control laws is randomized per subject. Four quantitative measures are used to compare the control laws: targeting error, settling time, overshoot and rise time. The targeting error is defined as the distance between the target location and the needle tip at the end of the insertion. Rise time is the time it takes to reach $95 \%$ of the target distance. The settling time is the time needed to reach and stay within an error band of $10 \%$ around the target.

Mauchly's sphericity test indicated that all quantitative measures violated the sphericity assumption (targeting error: $\chi^{2}(5)=36.774, \quad P<0.001$; overshoot: $\chi^{2}(5)=45.572, \quad P<0.001$; rise time: $\chi^{2}(5)=17.877$, $P=0.003$; settling time: $\left.\chi^{2}(5)=15.967, P=0.007\right)$. The repeated-measures ANOVA with a Greenhouse-Geisser correction shows that only the overshoot presents significant differences $\left(F_{(1.513,28.743)}=5.742, P=0.013\right)$, indicating that the damper control law scores significantly worse than the other three control laws. A representative result of one subject is presented in Fig. 6.

In addition to the quantitative evaluation, we also evaluate subjects' opinion. After the experiment, participants are asked to fill in a questionnaire to indicate how easy, intuitive, quick and accurate each control law is. The questionnaire contains a set of statements, where a score of 5 is described as "completely agree" and 1 as "completely disagree". The qualitative data does not violate the sphericity assumption based on Mauchly's sphericity test. Perceived easiness, intuitiveness and accuracy showed significant differences in repeated-measures ANOVA $\left(F_{(3,57)}=10.008, P<0.001 ; F_{(3,57)}=3.597\right.$, $P=0.019 ; F_{(3,57)}=9.814, P<0.001$, respectively). These results indicate that the damper control law is evaluated worse than the other three control laws. The answers regarding the control law of preference violates the sphericity assumption (Mauchly's sphericity test: $\left.\chi^{2}(5)=11.889, \quad P=0.037\right)$. The repeated-measures ANOVA shows that the squared velocity control law is significantly more preferred by the subjects than the other control laws $\left(F_{(2.195,41.705)}=5.783, P=0.002\right)$.

The results of the quantitative and qualitative measures are plotted in Fig. 5. Although the quantitative analysis does not indicate significant differences between the squared velocity, position and velocity control laws, the qualitative analysis shows that the squared velocity control law is preferred by users. The squared velocity control law is then selected to be used in the tele-operation scheme of the MIRIAM robot. This control law is used in the final experiments inserting the needle towards a physical target, which is detected by the pre-operative target detection.

\section{3. $\quad$ Target segmentation evaluation}

The proposed method is evaluated using the Dice similarity coefficient (DSC) [40]. The DSC measures the
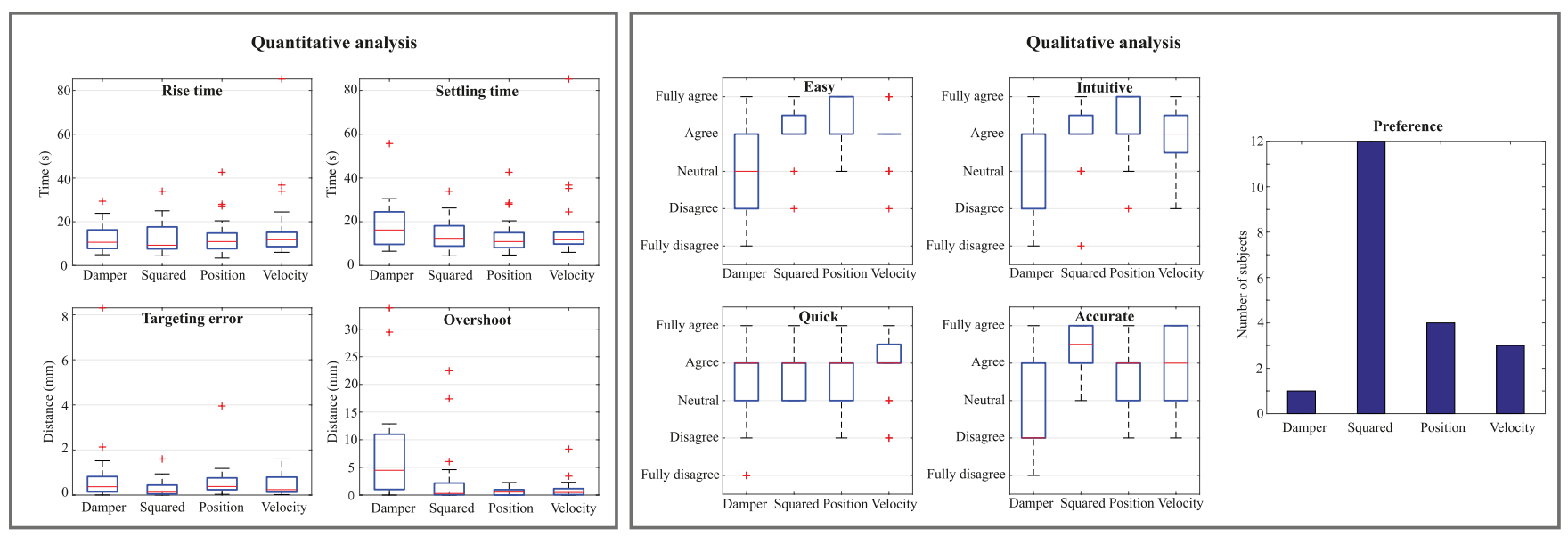

Fig. 5. Overall results of the statistic analysis of different insertion control laws. In the qualitative analysis, the damper control presents the highest overshoot, while the squared velocity presents the lowest targeting error. The qualitative analysis shows that the squared velocity control law is considered intuitive and the control law of preference. Based on the analysis, the squared velocity control law is chosen to be implemented in the MIRIAM robot. 


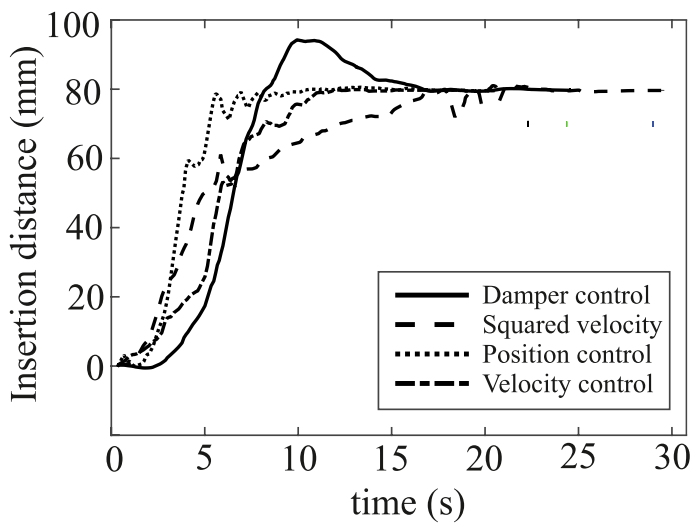

Fig. 6. A representative results of one subject performing insertions using the four control laws. The damper control law presents a significant overshoot, while the position control presents the best rise time.

similarity between two images, which ranges from 0 for no correspondence between the images to 1 for complete correspondence. The DSC is defined as

$$
\operatorname{DSC}=\frac{2\left|A_{a} \bigcap A_{m}\right|}{\left|A_{a}\right|+\left|A_{m}\right|}
$$

where $A_{a}$ is the target segmented by the algorithm and $A_{m}$ is the target manually segmented. According to the literature, an acceptable similarity occurs when DSC $>0.70$ [41]. The algorithm is evaluate using three different groups of MR images: (1) soft tissue phantoms, (2) biological phantom and (3) patient data.

\subsubsection{Soft tissue phantom}

A gelatin-based phantom is prepared with a mass ratio of $15 \%$ gelatin and $85 \%$ water. Spherical and cubical targets made of polyvinyl chloride (PVC) with dimensions ranging between 4 and $6 \mathrm{~mm}$ are embedded in the phantom. An MR scan of axial slices is performed using a T2 Turbo Spin Echo (TSE) imaging protocol. The slice thickness is set to $1.0 \mathrm{~mm}$ and field of view (FoV) of $200 \mathrm{~mm} \times 200 \mathrm{~mm}$. The average DSC from 15 different images is 0.79 with a standard deviation of 0.04 .

\subsubsection{Biological phantom}

Spherical and cubical targets with dimensions ranging between $4 \mathrm{~mm}$ and $6 \mathrm{~mm}$ are embedded in an ex-vivo prostate of a bull. The same imaging protocol from the previous group is used. The average DSC from 15 different images is 0.80 with a standard deviation of 0.05 .

\subsubsection{Patient data}

The proposed method is also evaluated in patient images. The MR images of 15 prostates available at the Cancer
Imaging Archive are used to evaluate the accuracy of the method in patient images [42]. A representative result in one patient image is presented in Fig. 7. The two-algorithm solution is important to avoid segmentation drifting, as can be seen in Fig. 7. The average DSC from the 15 different patient images is 0.77 with a standard deviation of 0.08 .

\subsubsection{Target location}

The center of the segmented target is computed for the slices where the suspected lesion is visible. The average of all centers is defined as the target location. In order to compute the target position with respect to the robot, the fiducial located at the robot needle guide is also detected by the algorithm. The fiducial is used to localize the robot with respect to the fixed frame of the MR scanner. Standard homogeneous transformations are used to calculate the target location with respect to the robot, which is used as an input to insert the needle towards the target.

\subsection{MRI experiments}

Tele-operated insertions are performed by four different subjects (Fig. 8). A pre-operative MR scan is performed before the experiments using a T2 Turbo Spin Echo (TSE) imaging protocol. The slice thickness is set to $3.0 \mathrm{~mm}$, field of view (FoV) of $200 \mathrm{~mm} \times 200 \mathrm{~mm}$, echo time of $1.01 \mathrm{~s}$, repetition time of $7.50 \mathrm{~s}$ and acquisition time of $4.5 \mathrm{~min}$. The two targets embedded in the soft tissue phantom are localized with respect to the robot reference frame. The targets are located at $(x=-13 \mathrm{~mm}, y=-6 \mathrm{~mm}, z=89 \mathrm{~mm})$ and $(x=$ $-18 \mathrm{~mm}, y=7 \mathrm{~mm}, z=89 \mathrm{~mm}$ ), respectively (Fig. 9). Each subject performs one insertion. No practice trials are allowed to also assess the difference in the level of user experience. The subjects are divided into

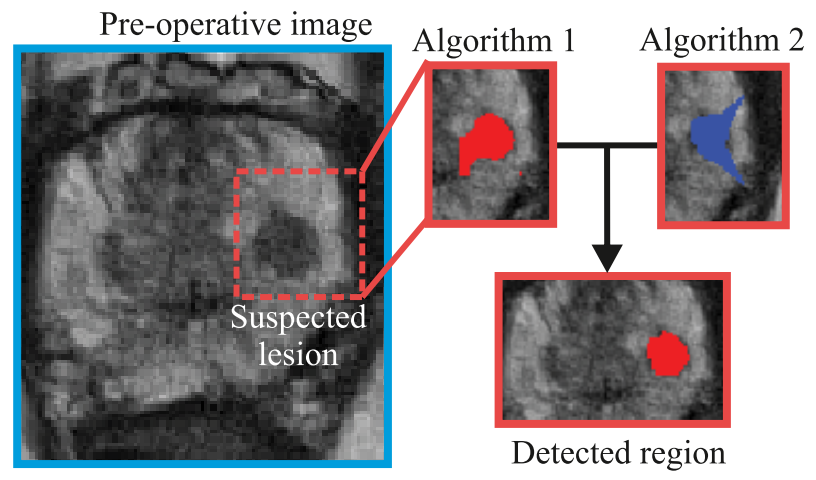

Fig. 7. One representative results of segmenting a suspected lesion in a patient image. Algorithm 1 is the region-dependent process, while Algorithm 2 is the edge-dependent. 


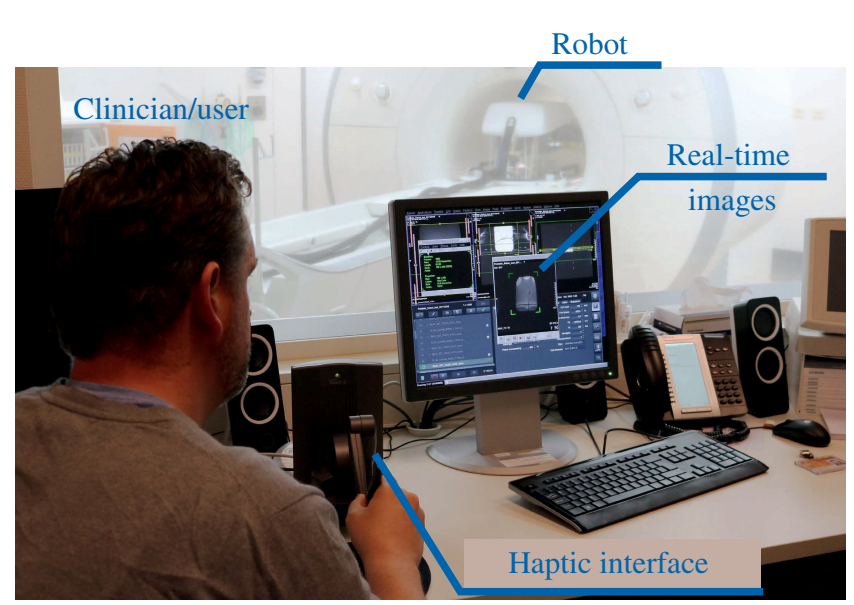

Fig. 8. The clinician controls the insertion via a haptic device located in the control room, while the robot is inside the Magnetic Resonance (MR) scanner. Real-time MR images are provided to the clinician, allowing the supervision of the procedure. Please refer to the accompanying video that demonstrates the experimental results.

three groups:

- Experienced users: Subjects who also participated in the experimental evaluation previously performed in Sec. 3.2.

- Intermediate users: Subjects who had performed up to five insertions using the system.

- Beginner users: Subjects who had never used a haptic device before.

After each insertion, two MR scans (axial and coronal using TSE imaging protocol, slice thickness is set to $1.0 \mathrm{~mm}$ and field of view (FoV) of $200 \mathrm{~mm} \times 200 \mathrm{~mm}$ ) are performed in order to calculate the targeting error. The targeting error is defined as the Euclidian distance between the needle tip and the center of the target. The errors along the $x$ - and $y$-axis are calculated using the axial slices, while the error along $z$ is calculated using the coronal slices. Please refer to the accompanying video that demonstrates the experimental results. The results of two representative experiments are shown in Fig. 9. The experienced users achieve an average targeting error of $1.9 \mathrm{~mm}$ in less than $22 \mathrm{~s}$. The intermediate user achieved a targeting error of $4.6 \mathrm{~mm}$, while the targeting error for the beginner user is $5.4 \mathrm{~mm}$. The beginner and intermediate users finished the insertion before the target was actually reached. The results suggest that the level of experience plays an important role in the targeting accuracy. However, it is important to mention that the number of subjects in the final experimental study limits our observations regarding the influence of user experience in targeting accuracy. Therefore, the learning curve of users and how user experience impacts the accuracy will be addressed in a future study with a larger number of subjects.

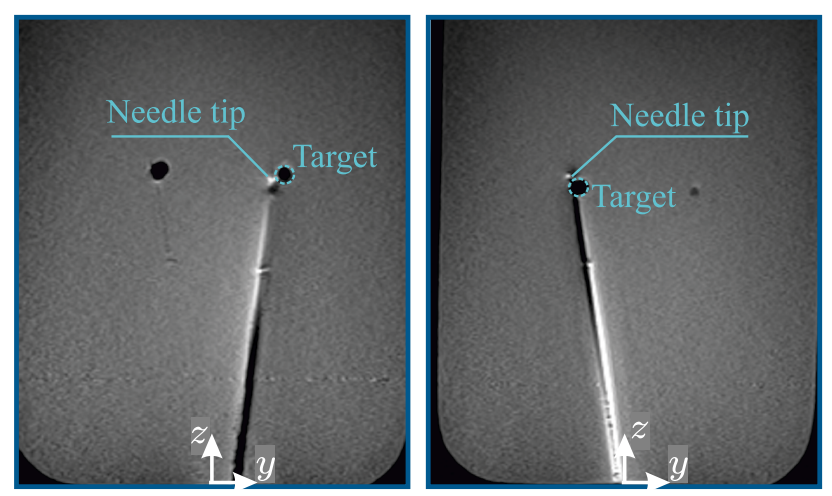

Fig. 9. Two representative insertions of the biopsy needle towards a target. The needle tip reaches the target with a targeting error between $1.7 \mathrm{~mm}$ (experienced user) and $5.4 \mathrm{~mm}$ (beginner user).

The targeting errors achieved in the insertions are within an acceptable range, i.e. in the range of clinically significant tumor size in pathology [43]. The targeting errors can be reduced using a closed-loop controller to perform axial needle rotations during the insertion. A closed-loop steering algorithm requires a needle tip tracker using real-time MR images. MR-based needle tip tracking is a challenging task and is considered beyond the scope of this work. However, it is important to highlight that insertion depth and needle rotations can be decoupled and implementing a closed-loop steering algorithm to control axial needle rotations is straightforward.

\section{Conclusions}

In this study, we present an MR-compatible robotic system for tele-operated needle-based interventions in the prostate. The user tele-operates a 9 DoF robot using a haptic device. Force feedback is provided to the user by a needle-tissue interaction force model. The parameters of the model are estimated using the data collected during 84 insertions. Human subject studies are presented to evaluate four different laws that relate user input into robot commands. The squared velocity law is shown to be the best control law for the MIRIAM tele-operated robot. Moreover, a targeting detection algorithm is proposed and evaluated using phantom and patient images. The suspected lesion (target) is detected in 45 images with DSC higher than 0.70 . The complete system is evaluated in four tele-operated insertions towards a target with an average targeting error of $3.4 \mathrm{~mm}$. Although users consider the system intuitive, the results indicate that previous practice with the system is important to reduce the targeting error. Therefore, the learning curve of users has to be investigated in future studies. Another ongoing work is the development of a 
path planner that takes into account the shape of the lesion to define the insertion direction that will maximize the amount of tissue collected from the suspected lesion.

Future work will focus on implementing a steering algorithm to control needle rotations during the insertion. Needle tip tracking using either Fiber Bragg Grating (FBG) sensors or real-time MR images will be integrated into the system. The steering algorithm will compensate deviations from the intended path, thus reducing the targeting error. In order to improve the needletissue force model, we will combine the model with FBG-based force sensing technique [44] to provide force feedback. This integration will allow us to study the user feedback perception as the needle crosses different tissue layers. Moreover, experiments in human cadavers and an extensive evaluation with clinicians are planned.

\section{Acknowledgments}

This research was supported by funds from the Dutch Ministry of Economic Affairs and the Provinces of Overijssel and Gelderland, within the Pieken in de Delta (PIDON) Initiative, Project MIRIAM.

\section{References}

1. J. Ferlay, I. Soerjomataram, R. Dikshit, S. Eser, C. Mathers, M. Rebelo, D. Parkin, D. Forman and F. Bray, Cancer incidence and mortality worldwide: Sources, methods and major patterns in globocan 2012, Int. J. Cancer 136(5) (2015) 359-386.

2. M. G. Schouten, J. G. Bomers, D. Yakar, H. Huisman, E. Rothgang, D. Bosboom, T. W. Scheenen, S. Misra and J. J. Fütterer, Evaluation of a robotic technique for transrectal MRI-guided prostate biopsies, Eur. Radiol. 22(2) (2012) 476-483.

3. P. Moreira, G. van de Steeg, T. Krabben, J. Zandman, E. E. G. Hekman, F. van der Heijden, R. J. H. Borra and S. Misra, Miriam robot: A novel robotic system for MR-guided needle insertion in the prostate, J. Med. Robot. Res. 2(3) (2017) 1750 006-1-1 750 006-13.

4. M. Abayazid, C. Pacchierotti, P. Moreira, R. Alterovitz, D. Prattichizzo and S. Misra, Experimental evaluation of co-manipulated ultrasound-guided flexible needle steering, Int. J. Med. Robot. 12(2) (2015) 219-230.

5. G. P. Moustris, S. C. Hiridis, K. M. Deliparaschos and K. M. Konstantinidis, Evolution of autonomous and semi-autonomous robotic surgical systems: A review of the literature, Int. J. Med. Robot. 7(4) (2011) 375-392.

6. N. Hungr, J. Troccaz, N. Zemiti and N. Tripodi, Design of an ultrasound-guided robotic brachytherapy needle-insertion system, in Proc. Annual Int. Conf. IEEE Engineering in Medicine and Biology Society, (2009), pp. 250-253.

7. O. Piccin, L. Barbé, B. Bayle, M. Mathelin and A. Gangi, A force feedback teleoperated needle insertion device for percutaneous procedures, Int. J. Robot. Res. 28(9) (2009) 1154-1168.

8. A. Majewicz and A. M. Okamura, Cartesian and joint space teleoperation for nonholonomic steerable needles, in Proc. World Haptics Conference (WHC), 2013, (2013), pp. 395-400.
9. J. M. Romano, R. J. Webster and A. M. Okamura, Teleoperation of steerable needles, in Proc. IEEE Int. Conf. Robotics and Automation, (2007), pp. 934-939.

10. N. Abolhassani and R. V. Patel, Teleoperated master-slave needle insertion, Int. J. Med. Robot. 5(4) (2009) 398-405 [Online]. Available at http://dx.doi.org/10.1002/rcs.269.

11. W. Zarrad, P. Poignet, R. Cortesao and O. Company, Towards teleoperated needle insertion with haptic feedback controller, in IEEE/RSJ Int. Conf. Intelligent Robots and Systems, (San Diego, CA, 2007), pp. 1254-1259.

12. Z. Wei, G. Wan, L. Gardi, G. Mills, D. Downey and A. Fenster, Robotassisted 3D-TRUS guided prostate brachytherapy: System integration and validation, Med. Phys. 31(3) (2004) 539-548.

13. D. Stoianovici, C. Kim, G. Srimathveeravalli, P. Sebrecht, D. Petrisor, J. Coleman, S. Solomon and H. Hricak, MRI-safe robot for endorectal prostate biopsy, IEEE/ASME Trans. Mechatronics 19(4) (2014) 1289-1299.

14. A. A. Goldenberg, J. Trachtenberg, Y. Yi, R. Weersink, M. S. Sussman, M. Haider, L. Ma and W. Kucharczyk, Robot-assisted MRI-guided prostatic interventions, Robotica 28(2) (2010) 215-234.

15. R. Seifabadi, F. Aalamifar, I. Iordachita and G. Fichtinger, Toward teleoperated needle steering under continuous MRI guidance for prostate percutaneous interventions, Int. J. Med. Robot. 12(3) (2016) 355-369.

16. A. M. Okamura, C. Simone and M. D. O'Leary, Force modeling for needle insertion into soft tissue, IEEE Trans. Biomed. Eng. 51(10) (2004) 1707-1716.

17. L. Barbé, B. Bayle and M. de Mathelin, In vivo model estimation and hapatic chacterization of needle insertions, Int. J. Robot. Res. 26 (2007) 1283-1301.

18. Y. Kobayashi, A. Onishi, H. Watanabe, T. Hoshi, K. Kawamura and M. G. Fujie, In vitro validation of viscoelastic and nonlinear physical model of liver for needle insertion simulation, in Proc. Int. Conf. Biomedical Robotics and Biomachatronics, (2008), pp. 469-476.

19. G. Ravali and M. Manivannan, Haptic feedback in needle insertion modeling and simulation: Review, IEEE Rev. Biomed. Eng. PP(99) (2017) 1-1.

20. L. Gong, S. D. Pathak, D. R. Haynor, P. S. Cho and Y. Kim, Parametric shape modeling using deformable superellipses for prostate segmentation, IEEE Trans. Med. Imag. 23(3) (2004) 340-349.

21. M. J. Costa, H. Delingette, S. Novellas and N. Ayache, Automatic segmentation of bladder and prostate using coupled 3D deformable models, in Int. Conf. Med. Imag. Comput. Comput. Assist. Intervention 2007, pp. 252-260.

22. M. Mazonakis, J. Damilakis, H. Varveris, P. Prassopoulos and N. Gourtsoyiannis, Image segmentation in treatment planning for prostate cancer using the region growing technique, $\mathrm{Br}$. J. Radiol. 74(879) (2001) 243-249.

23. A. Firjani, A. Elnakib, F. Khalifa, G. Gimel'farb, M. A. El-Ghar, J. Suri, A. Elmaghraby and A. El-Baz, A new 3d automatic segmentation framework for accurate segmentation of prostate from DCE-MRI, in Proc. IEEE Int. Symp. Biomedical Imaging: From Nano to Macro, (Chicago, IL, 2011), pp. 1476-1479.

24. S. Doyle, A. Madabhushi, M. Feldman and J. Tomaszeweski, A boosting cascade for automated detection of prostate cancer from digitized histology, in Int. Conf. Med. Imag. Comput. Comput. Assist. Intervention 2006, pp. 504-511.

25. S. Wang, K. Burtt, B. Turkbey, P. Choyke and R. M. Summers, Computer aided-diagnosis of prostate cancer on multiparametric MRI: A technical review of current research, Biomed. Res. Int. 2014 (2014) 789561.

26. S. Ozer, D. L. Langer, X. Liu, M. A. Haider, T. H. van der Kwast, A. J. Evans, Y. Yang, M. N. Wernick and I. S. Yetik, Supervised and unsupervised methods for prostate cancer segmentation with multispectral MRI, Med. Phys. 37(4) (2010) 1873-1883. 
27. Y. Guo, Y. Gao and D. Shen, Deformable MR prostate segmentation via deep feature learning and sparse patch matching, IEEE Trans. Med. Imaging 35(4) (2016) 1077-1089.

28. Y. Artan, D. L. Langer, M. A. Haider, T. H. van der Kwast, A. J. Evans, M. N. Wernick and I. S. Yetik, Prostate cancer segmentation with multispectral MRI using cost-sensitive conditional random fields, in Proc. IEEE Int. Symp. Biomedical Imaging: From Nano to Macro, (Boston, MA, 2009), pp. 278-281.

29. X. Liu, D. L. Langer, M. A. Haider, Y. Yang, M. N. Wernick and I. S. Yetik, Prostate cancer segmentation with simultaneous estimation of markov random field parameters and class, IEEE Trans. Med. Imag. 28(6) (2009) 906-915.

30. M. M. Mohamed, T. K. Abdel-galil, M. A. Salama, E. F. El-saadany, M. Kamel, A. Fenster, D. B. Downey and K. Rizkalla, Prostate cancer diagnosis based on gabor filter texture segmentation of ultrasound image, in Proc. Canadian Conf. Electrical and Computer Engineering. Toward a Caring and Humane Technology (Cat. No. 03CH37436), Vol. 3, (Montreal, Quebec, 2003), pp. 1485-1488.

31. H. Akbari, X. Yang, L. V. Halig and B. Fei, 3d segmentation of prostate ultrasound images using wavelet transform, Vol. 7962 (2011) $79622 \mathrm{~K}-79622 \mathrm{~K}-6$.

32. G. Litjens, R. Toth, W. van de Ven, C. Hoeks, S. Kerkstra, B. van Ginneken, G. Vincent, G. Guillard, N. Birbeck, J. Zhang, R. Strand, F. Malmberg, Y. Ou, C. Davatzikos, M. Kirschner, F. Jung, J. Yuan, W. Qiu, Q. Gao, P. E. Edwards, B. Maan, F. van der Heijden, S. Ghose, J. Mitra, J. Dowling, D. Barratt, H. Huisman and A. Madabhushi, Evaluation of prostate segmentation algorithms for MRI: The promise12 challenge, Med. Image Anal. 18(2) (2014) 359-373.

33. C. Richard, On the identification and haptic display of friction, Ph.D. dissertation, Stanford University (2000).

34. J. J. Abbott and A. M. Okamura, Virtual fixture architectures for telemanipulation, in Proc. IEEE Int. Conf. Robotics and Automation, Vol. 2, (IEEE, Taipei, 2003), pp. 2798-2805.

35. N. Otsu, A threshold selection method from gray-level histograms, IEEE Trans. Syst. Man. Cybern. Syst. 9(1) (1979) 62-66.

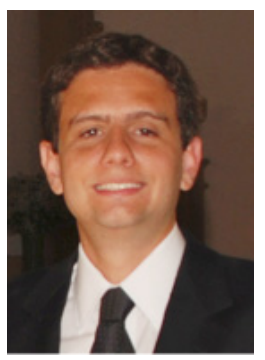

Pedro Moreira is currently a research fellow at the Harvard Medical School and the Brigham and Women's Hospital. He was a postdoctoral fellow at the University of Twente (The Netherlands) between 2013 and 2017. He obtained his MSc degree in Electrical Engineering from the Federal University of Rio de Janeiro (Brazil). He received his PhD degree in Automatic Systems and Microelectronics from the University of Montpellier (France) in 2012. Before starting his $\mathrm{PhD}$, he worked for six years at the Electric Energy Research Center in Brazil. His main research interests are surgical robotics, flexible needle steering and control theory.

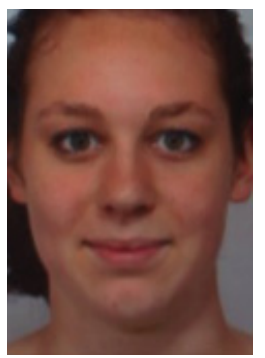

Leanne Kuil received the B.Sc. degree in Biomedical Technology and the M.Sc. degree in Biomedical Engineering from the University of Twente, Enschede, The Netherlands, in 2013 and 2016, respectively. She worked on her M.Sc. degree project within the Surgical Robotics Laboratory, which focused on tele-operated needle steering and MRI-compatible robots. She is currently a consultant at Kaylane BV, Utrecht, The Netherlands.
36. R. J. Roesthuis, M. Kemp, J. J. van den Dobbelsteen and S. Misra, Three-dimensional needle shape reconstruction using an array of fiber bragg grating sensors, IEEE/ASME Trans. Mechatronics 19(4) (2014) 1115-1126.

37. M. B. Boubaker, M. Haboussi, J.-F. Ganghoffer and P. Aletti, Finite element simulation of interactions between pelvic organs: Predictive model of the prostate motion in the context of radiotherapy, J. Biomech. 3(137) (2009) 1862-1868.

38. W. Assaad and S. Misra, Combining ultrasound-based elasticity estimation and $\{\mathrm{FE}\}$ models to predict $3 \mathrm{~d}$ target displacement, Med. Eng. Phys. 35(4) (2013) 549-554.

39. P. Moreira, N. Zemiti, C. Liu and P. Poignet, Viscoelastic model based force control for soft tissue interaction and its application in physiological motion compensation, Comput. Methods Programs Biomed. 116(2) (2014) 52-67.

40. Y. Yaegashi, K. Tateoka, K. Fujimoto, T. Nakazawa, A. Nakata, Y. Saito, T. Abe, M. Yano and K. Sakata, Assessment of similarity measures for accurate deformable image registration, J. Nucl. Med. Radiat. Ther. 42(12) (2012).

41. A. P. Zijdenbos, B. M. Dawant, R. A. Margolin and A. C. Palmer, Morphometric analysis of white matter lesions in MR images: Method and validation, IEEE Trans. Med. Imag. 13(4) (1994) 716724.

42. Data from prostate-diagnosis. the cancer imaging archive, 2015, http://doi.org/10.7937/K9/TCIA.2015.FOQEUJVT.

43. T. A. Stamey, F. S. Freiha, J. E. McNeal, E. A. Redwine, A. S. Whittemore and H. P. Schmid, Localized prostate cancer. Relationship of tumor volume to clinical significance for treatment of prostate cancer, Cancer, 71(S3) (1993) 933-938.

44. F. Khan, R. J. Roesthuis and S. Misra, Force sensing in continuum manipulators using fiber bragg grating sensors, in Proc. IEEE Int. Conf. Intelligent Robots and Systems (IROS), Vancouver, Canada, 24-28 September 2017, p. 2531-2536.

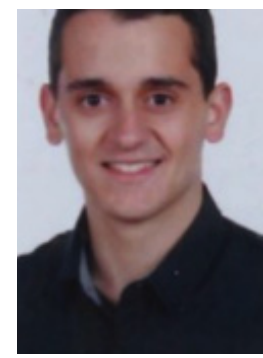

Pedro Dias received the B.Sc. degree in Biomedical Engineering from the New University of Lisbon, Portugal. He's currently working toward an M.Sc. degree also in Biomedical Engineering at New University of Lisbon. He was a visiting Master student at the University of Twente, working in the Surgical Robotics Laboratory in 2016.

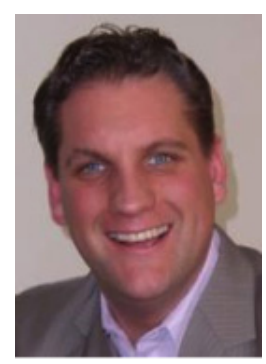

Ronald Borra received his PhD. in Experimental Radiology from the Turku University (Finland) in 2009 and his M.D. degree from the University of Groningen (Netherlands) in 2006. He was with the Harvard Medical School from 2010 to 2015. He is currently an Associate Professor of Nuclear Medicine \& Molecular Imaging at the University of Groningen, and Adjunct Professor of Experimental Radiology at the University of Turku. His research interest includes advanced magnetic resonance imaging (MRI) of cancer and positron emission tomography (PET) imaging. 


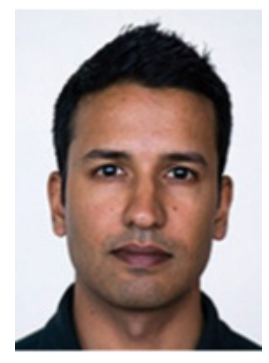

Sarthak Misra joined the University of Twente in 2009. He is currently a Full Professor in the Department of Biomechanical Engineering within the Faculty of Engineering Technology. He directs the Surgical Robotics Laboratory, and is affiliated with MIRA - Institute for Biomedical Technology and Technical Medicine. He is also affiliated with the Department of Biomedical Engineering, University of Groningen and University Medical Center Groningen. Baltimore, USA. Prior to commencing his studies at Johns Hopkins, he worked for three years as a dynamics and controls analyst at MacDonald Dettwiler and Associates on the International Space Station
Program. Sarthak received his Master of Engineering degree in Mechanical Engineering from McGill University, Montreal, Canada. He is the recipient of the European Research Council (ERC) Starting and Proof-ofConcept grants, Netherlands Organization for Scientific Research (NWO) VENI and VIDI awards, and NASA Space Flight Awareness award. He is the co-chair of the IEEE Robotics and Automation Society Technical Committee on Surgical Robotics and area co-chair of the IFAC Technical Committee on Biological and Medical Systems. Sarthak's broad research interests are primarily in the area of applied mechanics at both macro and micro scales. He is interested in the modeling and control of electro-mechanical systems with applications to medical robotics. 